\title{
Evidence for an antagonist form of the chemokine CXCL10 in patients chronically infected with HCV
}

\begin{abstract}
Armanda Casrouge,1,2 Jérémie Decalf,, ${ }^{1,2}$ Mina Ahloulay, ${ }^{3,4,5}$ Cyril Lababidi, ${ }^{6}$ Hala Mansour, ${ }^{1}$ Anaïs Vallet-Pichard,3,4,5 Vincent Mallet, ${ }^{3,4,5}$ Estelle Mottez, ${ }^{6}$ James Mapes, ${ }^{7}$ Arnaud Fontanet, ${ }^{8}$ Stanislas Pol, ${ }^{3,4,5}$ and Matthew L. Albert ${ }^{1,2}$

${ }^{1}$ The Laboratory of Dendritic Cell Biology, Department of Immunology, Institut Pasteur, Paris, France. ${ }^{2}$ INSERM U818, Paris, France. ${ }^{3}$ Université Paris Descartes, Paris, France. ${ }^{4}$ Institut Cochin, INSERM (IMR-S1016), CNRS (UMR 8104), Paris, France.

${ }^{5}$ Assistance Publique - Hôpitaux de Paris (APHP), Groupe Hospitalier Cochin Saint-Vincent de Paul, Unité d'Hépatologie, Paris, France. ${ }^{6}$ Centre for Human Immunology, Department of Immunology, Institut Pasteur, Paris France. ${ }^{7}$ Rules-Based Medicine, Austin Texas, USA ${ }^{8}$ Epidemiology of Emerging Infectious Diseases, Institut Pasteur, Paris, France.
\end{abstract}

\begin{abstract}
Chronic infection with hepatitis $\mathrm{C}$ virus (HCV) is a major public health problem, with nearly 170 million infected individuals worldwide. Current treatment for chronic infection is a combination of pegylated IFN- $\alpha_{2}$ and ribavirin (RBV); however, this treatment is effective in fewer than $50 \%$ of patients infected with $\mathrm{HCV}$ genotype 1 or 4. Recent studies identified the chemokine CXCL10 (also known as IP-10) as an important negative prognostic biomarker. Given that CXCL10 mediates chemoattraction of activated lymphocytes, it is counterintuitive that this chemokine correlates with therapeutic nonresponsiveness. Herein, we offer new insight into this paradox and provide evidence that CXCL10 in the plasma of patients chronically infected with HCV exists in an antagonist form, due to in situ amino-terminal truncation of the protein. We further demonstrated that dipeptidyl peptidase IV (DPP4; also known as CD26), possibly in combination with other proteases, mediates the generation of the antagonist form(s) of CXCL10. These data offer what we believe to be the first evidence for CXCL10 antagonism in human disease and identify a possible factor contributing to the inability of patients to clear HCV.
\end{abstract}

\section{Introduction}

There are nearly 170 million HCV-infected individuals worldwide (1). Many studies have explored the mechanisms by which the immune system is capable of mediating viral clearance (2). Although an $\mathrm{HCV}$-specific humoral response is commonly seen, $\mathrm{HCV}$-specific antibodies are not thought capable of conferring protection (3). Instead, protection in chimpanzees has been shown to correspond with $\mathrm{HCV}$-reactive $\mathrm{CD}^{+} \mathrm{T}$ cell responses (2). Similarly, in humans, tetramer analysis of successful resolvers indicates the presence of a high frequency of functional HCV-specific $\mathrm{CD}^{+} \mathrm{T}$ cells (4). In addition, there are genetic data to support a role for NK cells, based on the higher likelihood of KIR2DL3 and HLA-C1 individuals to clear infection (5). A specific and unique issue for chronic infection is that cure is possible, as $40 \%-80 \%$ of chronically infected patients (numbers vary depending on viral genotype) that receive pegylated IFN- $\alpha_{2}$ (peg-IFN- $\left.\alpha_{2}\right) / \mathrm{RBV}$ therapy clear the virus and achieve a sustained virologic response (SVR) (6). Still for many, the virus manages to circumvent natural immunity and existing therapeutic strategies, thus resulting in significant morbidity and mortality.

To better define the distinct clinical outcomes of HCV infection, many investigators have performed candidate molecule screens or transcriptional profiling in order to identify correlates of viral clearance. One molecule that has gained significant attention is CXCL10 (also known as IFN- $\gamma$-induced protein-10, or IP-10), an IFN- and TNF- $\alpha$-inducible chemokine that can be highly

Conflict of interest: James Mapes is an employee of Rules-Based Medicine Inc. Citation for this article: JClin Invest. 2011;121(1):308-317. doi:10.1172/JCI40594. expressed by endothelial cells, keratinocytes, fibroblasts, mesangial cells, astrocytes, monocytes, neutrophils, and hepatocytes (7). In addition to HCV disease, it has been shown to be expressed in many Th1-type inflammatory diseases, often correlating with target organ infiltration by $\mathrm{T}$ cells $(8,9)$. CXCL10 is part of a family of $\alpha$-chemokines that bind CXCR3, which also includes CXCL9 (also known as monokine induced by IFN- $\gamma$, or MIG) and CXCL11 (also known as IFN-inducible T cell $\alpha$ chemoattractant, or I-TAC). While all three ligands are induced by IFN and bind the same receptor, there now exist substantial data to support their unique roles in disease pathogenesis. This is clearly evident in chronic $\mathrm{HCV}$, where elevated levels of all three have been demonstrated but only CXCL10 is predictive of response to therapy (10).

Regarding published data, at least four independent studies have demonstrated that baseline levels of CXCL10 are predictive of the failure to respond to HCV treatment (10-13). Specifically, this has been demonstrated in patients with genotype 1 and $4 \mathrm{HCV}$; and in the largest study, a negative predictive value of $79 \%$ was reported for genotype 1 patients (12). In addition, elevated levels of CXCL10 have been reported in patients coinfected by $\operatorname{HCV} / \operatorname{HIV}(14,15)$; and with HCV-associated cryoglobulinemia (16). In several of these reports, CXCL10 levels correlated with necroinflammation, fibrosis stage, and/or HCV viral load. However, none of the reports have resolved the paradox of why a proinflammatory chemokine, responsible for recruiting activated lymphocytes to the liver, is a negative prognostic marker for response to therapy.

We hypothesized that CXCL10 may be present in an antagonist form in patients with chronic HCV, thus accounting for its correlation with treatment failure. To understand this prediction, 
some additional background is required. Briefly, chemokines act by binding to $G$ protein-coupled receptors and mobilization of intracellular $\mathrm{Ca}^{2+}$, which results in receptor internalization and the initiation of signaling pathways that facilitate chemotaxis. For the CXC class of chemokines, binding to and activation of the receptor is thought to be a two-step process. First, the core of the ligand binds the outer surface of the receptor; a second step involving the re-orientation of the flexible $\mathrm{N}$-terminal tail of the protein allows binding to a distinct domain within the receptor (17). Postsecretion modification of CXCL10 has been described, including C-terminal cleavage by MMP9 (or gelatinase B) and citrullination by peptidylarginine deiminase (PAD), both of which leave the protein in an agonist state $(18,19)$. Also reported is the $\mathrm{N}$-terminal cleavage of 2 amino acids by members of the X-prolyl dipeptidyl peptidase (DPP) family, the most characterized being dipeptidyl peptidase IV (DPP4 or CD26) (20). DPP4 has been shown to cleave several chemokines, including members of the $\alpha$-chemokine family $(21,22)$. Importantly, DPP4 truncation of CXCL10 generates a dominant negative form of the protein, which is capable of binding CXCR3 but does not induce signaling. Pertinent in vivo examples of chemokine antagonism include the degradation of monocyte chemoattractant protein-3 (MCP3) by MMP2, reported to be relevant to the pathogenesis of spondyloarthritis (23); and the cleavage of stromal cell-derived factor-1 (SDF1 or CXCL12) in HIV-associated neurodegeneration, also mediated by MMP2 (24). To date, there has been no in vivo evidence to support a role for CXCL10 antagonism in disease pathogenesis. In part, the challenge in establishing in vivo evidence is due to the inability to discriminate the different forms of the analytes using sensitive immune-based assays. Furthermore, few studies take care to exclude the activity of proteolytic plasma enzymes that may act extracorporeally on protein biomarkers of interest.

Herein, we show a correlation between CXCL10 levels and the precursor frequency of $\mathrm{CXCR} 3^{+}$cells in circulation, suggesting that the chemokine gradient in vivo is nonfunctional. This initial observation prompted us to investigate MMP and DPP family members as mediators of the inactivation of CXCL10, and our data suggest a potential in vivo role for DPP4. To support such studies, we have generated antibody-based assays that have been Clinical Laboratory Improvement Amendments (CLIA) certified and help to provide direct evidence that the $\mathrm{NH}_{2}$-terminal residues of plasma CXCL10 are cleaved in patients with chronic HCV. Following from our observations, we believe we have begun to unravel what has been a paradox in the field for several years - CXCL10 is elevated in nonresponders (NRs) and is in an antagonist form. Furthermore, our data suggest an important link between the metabolic complications and the chronic inflammation seen in $\mathrm{HCV}$ patients.

\section{Results}

Plasma CXCL10 levels correlate with high frequency of circulating CXCR3 ${ }^{+}$ cells in HCV patients. In order to identify biomarkers for response to therapy, we analyzed chronic HCV patients with plasma samples taken prior to, during, and following their 48-week course of peg-IFN- $\alpha_{2} / \mathrm{RBV}$. Multi-analyte profiling was performed on 84 selected molecules involved in inflammation, liver function, and metabolism. We performed Kruskal-Wallis test, comparing patients who failed to respond to therapy (NRs, $n=9$ ), those who cleared the virus (sustained virologic responders [SVRs], $n=13$ ), and healthy individuals $(n=7)$ (Figure 1 and Supplemental Table 1; supplemental material available online with this article;
doi:10.1172/JCI40594DS1). Of the 84 analytes, only CXCL10 and EGF showed a statistically significant difference between the NRs and SVRs at the pretherapy time point, using a Mann-Whitney $U$ test with a false discovery rate (FDR) correction (Figure 1A). Eleven other analytes were found to distinguish $\mathrm{HCV}$-infected patients from healthy donors, with most of them being involved in liver inflammation ( $\alpha-2 \mathrm{M}$, ferritin, fibrinogen, thyroid binding globulin, IL-18, ICAM, and sex hormone binding globulin [SHBG]) (25-28) and tissue remodeling (tissue inhibitor of metalloproteinases [TIMP] and VCAM-1) (29). While CXCL10 was elevated before therapy in NRs, levels were reduced during the course of therapy, again returning to high plasma levels 6 months after therapy (Figure 1B). In contrast to CXCL10, we observed no differences in the plasma concentration of CXCL9, a second CXCR3 ligand, nor did we see differences in CCL3 or CCL4, two ligands for CCR5, with the latter molecule also being an interferon-induced chemokine.

To gain insight into what may be occurring in vivo, we evaluated the fate of $\mathrm{CXCR}^{+}$cells in a small series of NRs who had high levels of plasma CXCL10. Based on the data of Zeremski et al., we assume that CXCL10 is principally produced by hepatocytes in patients chronically infected by HCV (30). This might suggest that CXCR3-expressing cells would selectively home to the liver. Alternatively, high levels of CXCL10 might disrupt the gradient and trigger CXCR3 receptor internalization. In either case, we predicted that in those patients with high plasma CXCL10, there would be few $\mathrm{CXCR}^{+}$cells in circulation. To test this prediction, we isolated PBMCs from 6 chronic NRs and $6 \mathrm{HCV}$ patients who achieved virologic response to therapy (SVRs), and determined the precursor frequency of $\mathrm{CXCR} 3^{+}$cells by cytometric analysis. Representative FACS plots are shown (Figure 2A), and the percentage of $\mathrm{CXCR}^{+}$cells among total $\mathrm{CD} 19^{+}$cells is reported for all patients (Figure 2B). Healthy donors were used as a control, showing a range of CXCR3-expressing cells of 8\%-19\%. Strikingly, we found a significantly higher frequency of CXCR $3^{+}$cells in $\mathrm{HCV}$ NRs, the same population with high plasma CXCL10 (Figure 2C). Prior studies supporting our findings found a higher frequency of $\mathrm{CXCR}^{+}$cells in chronic HCV patients to be restricted to B cells but not T cells (10); and a more recent article demonstrated higher levels of $\mathrm{CXCR}^{+} \mathrm{CD}^{+} \mathrm{T}$ cells (14). Regardless of the specific populations implicated, the data in Figures 1 and 2 suggested that plasma CXCL10 in chronic HCV patients may exists in a modified but still immunoreactive form (i.e., detectable by ELISA). This hypothesis was based on the absence of CXCR3 receptor internalization; and the fact that $\mathrm{CXCR} 3^{+}$cells are not being effectively drawn out of the circulation.

Establishing specific tools for monitoring CXCL10 chemokine antagonism. There exist 5 enzymes with X-prolyl DPP activity, including DPP2, DPP4, DPP8, DPP9, and FAP (20). Indeed, CXCL10 has been shown to be a substrate of DPP4, DPP8, and FAP $(22,31)$. DPP4 is a membrane protein that may be shed upon inflammation (referred to as sDPP4) (32). In addition, several chemokines are substrates for MMPs. Regarding CXCL10, it may be cleaved at its C-terminus by MMP9 and MMP12 (18, 33); however, the cleavage product produced remains an agonist for CXCR3 (18). One important and biologically relevant unknown is the action of MMP2 on CXCL10. Indeed, MMP2 has been shown to cleave the N-terminal amino acids of MCP1 and SDF1, thus generating an antagonist form of these molecules.

To evaluate the enzymes potentially implicated in cleavage of CXCL10, we first generated new tools for monitoring the agonist 
A

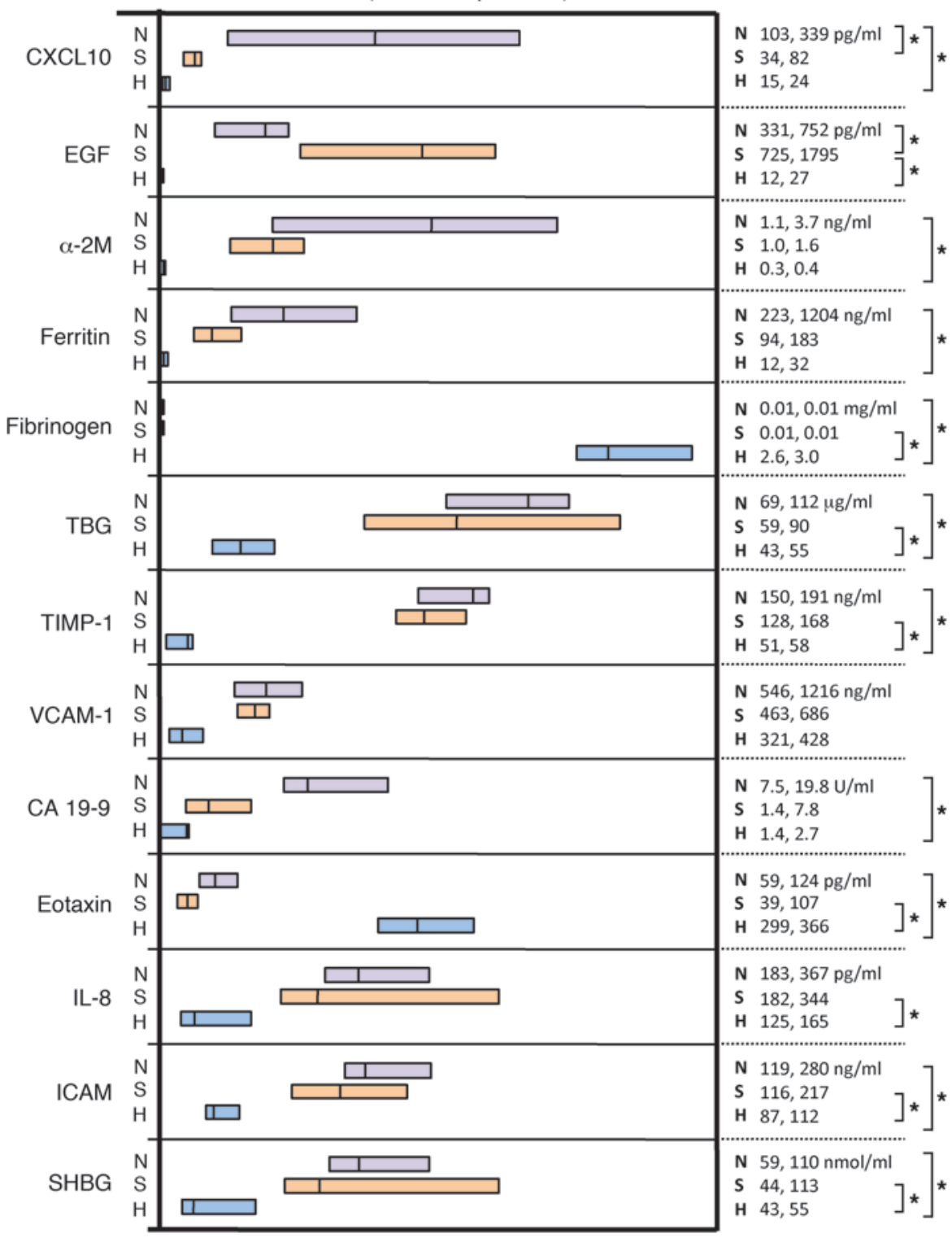

Figure 1

Multi-analyte profiling of chronic HCV patients confirms CXCL10 as a negative predictor for response to therapy. (A) Patient plasma was obtained prior to initiation of treatment and subjected to multianalyte profiling. Kruskal-Wallis test, comparing patients who failed to respond to therapy (NRs [N], $n=9$ ), those who cleared the virus (SVRs [S], $n=13$ ), and healthy individuals $(\mathrm{H}, n=7)$ revealed 13 analytes that distinguish the groups; *FDR-corrected $U$ test, $P<0.01$. ${ }^{\text {B }}$ Box plots indicate interquartile range 1 (IQR1) and IQR3, with vertical lines indicating median. Details for the multi-analyte profiling can be found in Supplemental Tables 1 and 2. (B) For the patients described in $\mathbf{A}$, data for 4 chemokines are shown over the kinetic course of treatment. Black circles, treatment NRs; gray squares, SVRs. ${ }^{*} P<0.01$, MannWhitney test with FDR correction. Pre-tx, before treatment.
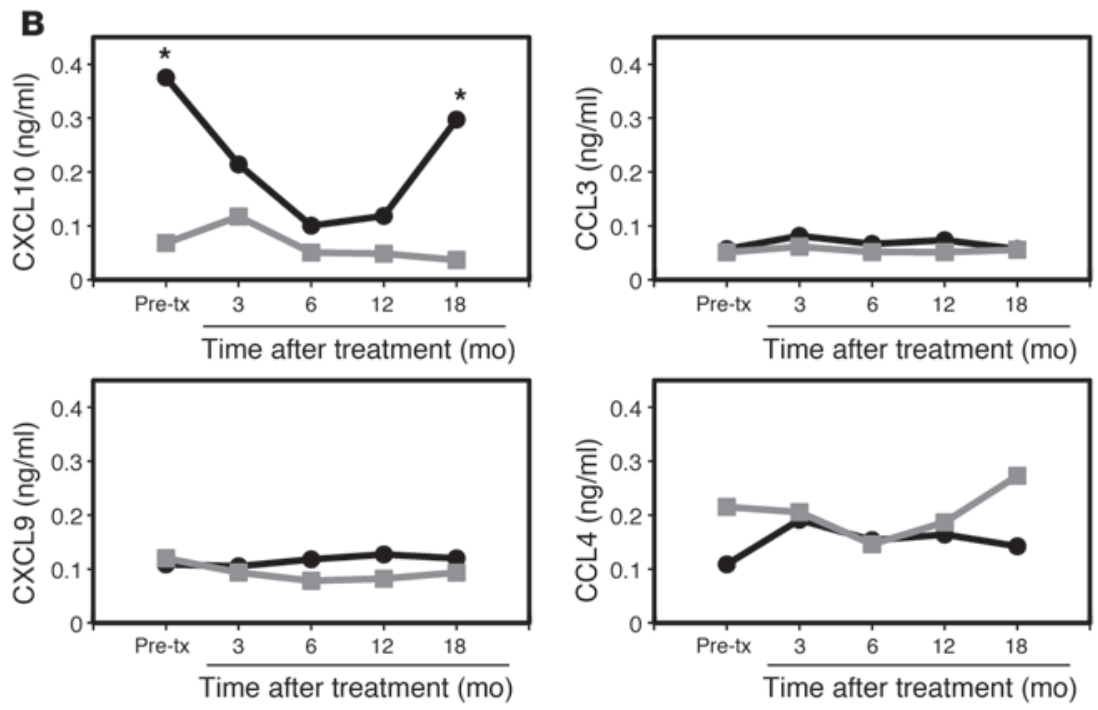
A
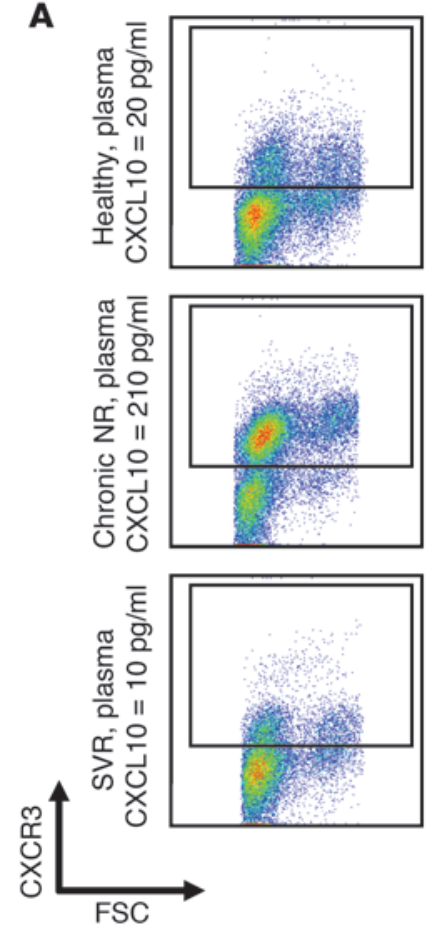

B

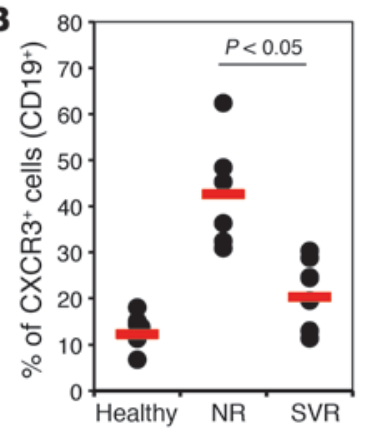

C

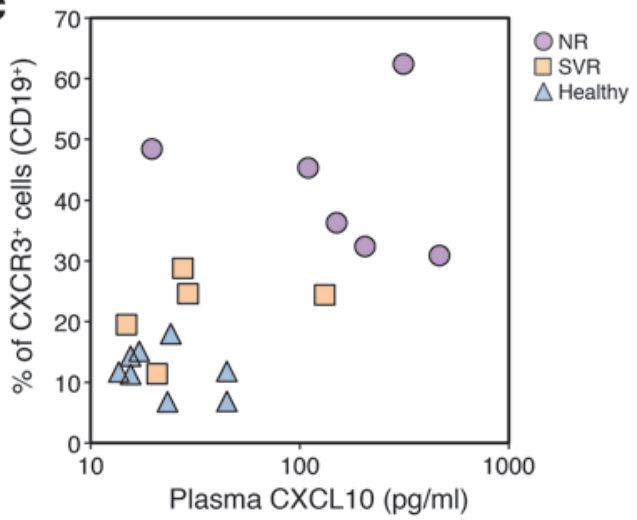

\section{Figure 2}

High frequency of $\mathrm{CXCR}^{+}$cells in NRs suggests that plasma CXCL10 is not bioactive. (A and $\mathbf{B}$ ) PBMC profiling studies were performed on chronic NRs, SVRs, and healthy individuals to determine the circulating levels of $\mathrm{CXCR}^{+}$ cells. Representative FACS plots are shown with the corresponding plasma concentration of total CXCL10 (A). Data from all groups are shown, with each filled circle representing a single individual; the red horizontal bars represent the median level of CXCR3-expressing CD19 $^{+}$cells (B). (C) Data from the same patients were plotted against the plasma concentration of total CXCL10. and N-terminal truncated forms of CXCL10. Specifically, we generated antibodies capable of distinguishing the agonist form of CXCL10 (1-77 aa). Rabbits were immunized with the $\mathrm{NH}_{2}$-terminal peptide, and immune serum was depleted of its activity to the truncated form using an immuno-adsorption strategy (see Methods for details). The polyclonal antiserum, termed L3, was able to detect recombinant CXCL10 (1-77 aa) but not $\mathrm{NH}_{2}$-terminal-truncated CXCL10 (3-77 aa). For monitoring $\mathrm{NH}_{2}$-trunctated CXCL10, we focused on the cleavage product created by DPP activity. In vitro selection from a library of single-chain antibodies resulted in the identification of clones capable of detecting the agonist form but not the truncated molecule; as well as reagents specific for the neo-epitope created upon cleavage of the $2 \mathrm{NH}_{2}$-terminal amino acids. Based on these screens, clone 10.1 for the long form and clone 52.1 for the short form were chosen. Detection assays were established, and a CLIA-certified 3-plex for Luminex was validated (Supplemental Figure 1 and data not shown).

Next, we exposed full-length recombinant CXCL10 to active MMP2, MMP9, and DPP4. As predicted, MMP9 cleaved 9 amino acids from CXCL10 (Figure 3A); and assaying for the $\mathrm{NH}_{2}$-terminal residues using anti-CXCL10 antiserum L3, we determined that MMP9 would be an unlikely candidate for generating an in vivo antagonist form of CXCL10 (Figure 3B). MMP2 did not alter the mass of CXCL10 (Figure 3A), and it also remained sensitive to antiserum L3. Finally, we used DPP4, which cleaved 2 amino acids from the $\mathrm{NH}_{2}$-terminus, confirmed by mass analysis and the generation of a neo-epitope that was recognized by antiCXCL10 clone 52.1 but not antiserum L3 (Figure 3B) or by the mAb clone 10.1 (data not shown).

To validate that our reagents are capable of detecting biologic forms of CXCL10, we recruited $5 \mathrm{HCV}$ patients initiating peg-IFN- $\alpha_{2} /$ RBV therapy. Whole blood was collected in BD P700 tubes, which contain DPP4 inhibitors, thus preventing extracorporeal cleavage. Additionally, we obtained heparinized blood for monitoring the frequency of CXCR3 cells in circulation. In all 5 study subjects, plasma CXCL10 was induced; however, the level of agonist CXCL10 differed among the individuals studied (Casrouge and Albert, unpublished observations). Data from one of the patients is shown in order to provide evidence that during peak CXCL10 induction, the agonist form may be detected (Figure 4A). Interestingly, we also observed that IFN treatment resulted in the disappearance of CXCR3-expressing cells from the circulation (Figure 4B). To evaluate the correlation between CXCL10 and circulating levels of $\mathrm{CXCR}^{+}$cells, we plotted the plasma concentration of CXCL10 (Figure 4C, black line) and compared it with the percentage of circulating $\mathrm{CXCR}^{+}$cells within the PBMCs (Figure $4 \mathrm{C}$, orange line). As shown, there was an indirect correlation between these parameters, consistent with CXCL10 acting as an agonist and mediating migration of CXCR3-expressing cells. This is in contrast to the relationship shown in nonstimulated chronic HCV patients (Figure 2C). Furthermore, analysis of absolute cell numbers indicated a marked decrease in the level of circulating $\mathrm{CXCR}^{+}$cells (Figure 4D, 35\% pretreatment at $2 \% 6$ hours after injection of peg-IFN- $\alpha_{2}$ - a $96 \%$ decrease as compared with a $16 \%$ decrease in CXCR3- cells). Our interpretation is that IFN treatment resulted in agonist CXCL10 production and migration of the $\mathrm{CXCR}^{+}$cells. Note that receptor internalization cannot be ruled out as contributing to the absence of detectable $\mathrm{CXCR}^{+}$ cells. Regardless of the mechanism, we demonstrate an important correlation between the presence of the agonist form of CXCL10 and decreased frequency of $\mathrm{CXCR}^{+}$cells in circulation. These data validate our CXCL10 assays as well as supporting the conclusion of the in vivo analysis of circulating $\mathrm{CXCR}^{+}$cells in relation to CXCL10 concentration (Figure 2). 
A
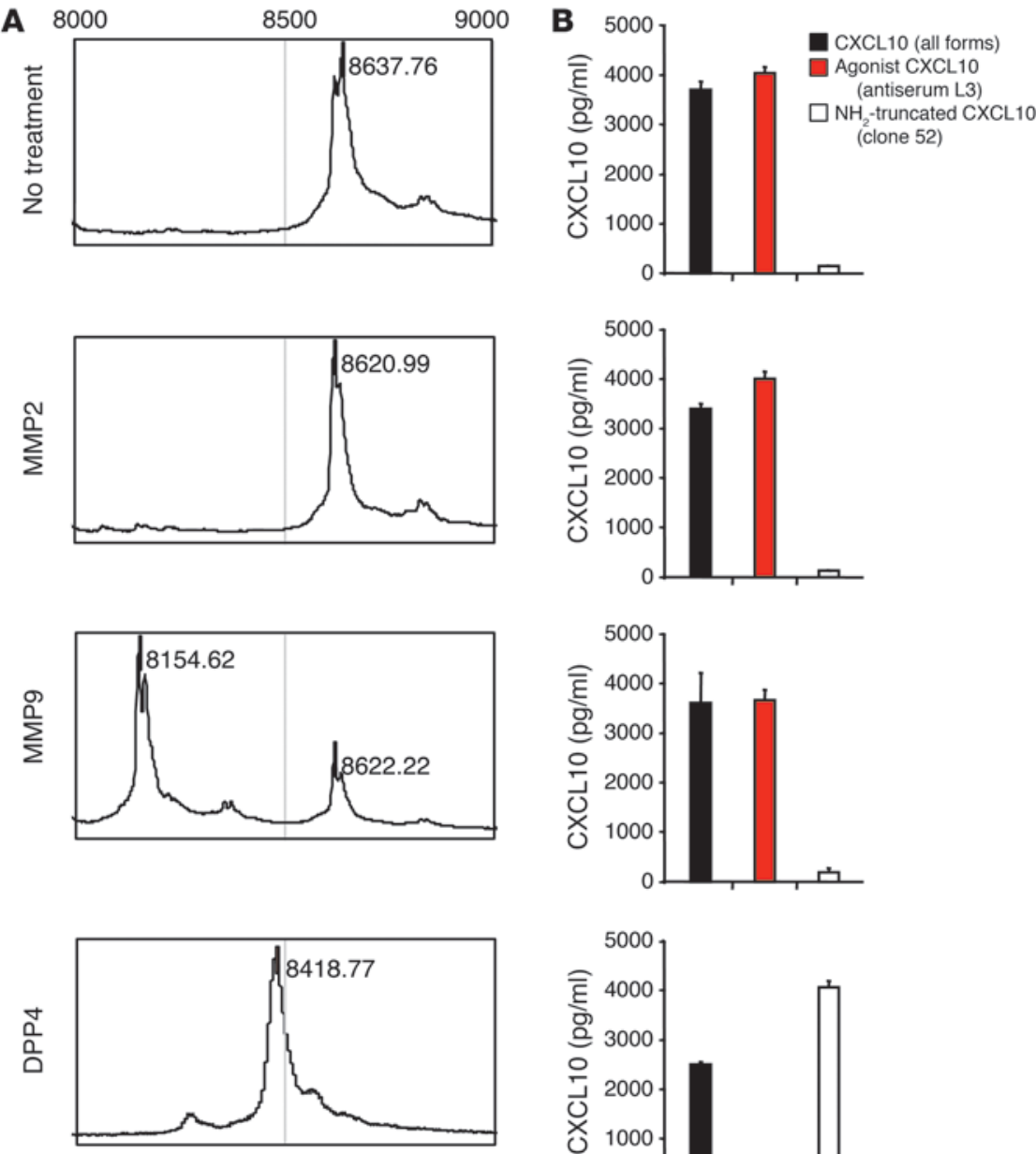
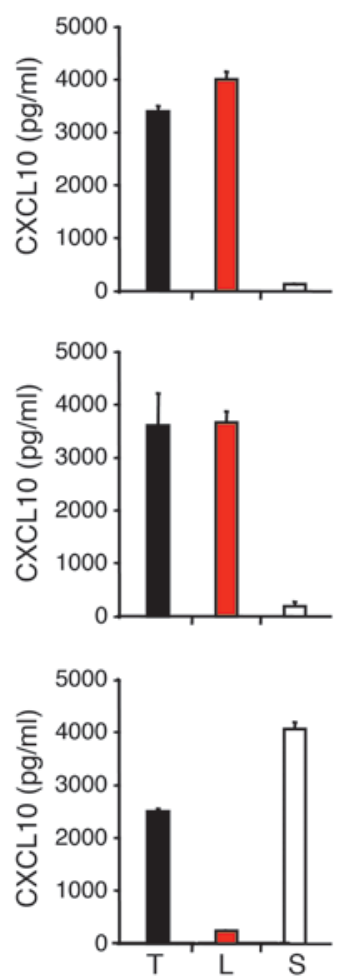

\section{Figure 3}

DPP4 but not MMP2 or MMP9 cleavage results in $\mathrm{NH}_{2}$-terminal truncation of CXCL10. Human recombinant CXCL10 (1-77 aa) was digested in vitro with MMP2, MMP9, or DPP4, as described in Methods. (A) $200 \mathrm{ng}$ of the digestion was spotted in an $\mathrm{H} 4$ array and analyzed by SELDITOF. Values are shown in daltons. (B) CXCL10 exposed to MMP2, MMP9, and DPP4 was run in 3 parallel ELISA assays to determine the relative percentage of total protein ( $T$, black bars) that is present in the agonistic long form (L, red bars) or $\mathrm{NH}_{2}$-truncated short form (S, white bars). Bars represent the mean of duplicate wells, and error bars indicate $1 \mathrm{SD}$ of the mean. Data are representative of 4 independent experiments.
Elevated DPP activity in chronic HCV patients is due to DPP4. To further investigate a potential mechanism for CXCL10 cleavage, we assayed plasma DPP activity in patients with $\mathrm{HCV}$ infection. Indeed, the plasma DPP activity was higher in chronic NRs as compared with SVRs and healthy individuals (Figure 5A). Using a tripeptide, broad-spectrum X-prolyl DPP inhibitor, isoleucine-proline-isoleucine (IPI), it was possible to inhibit more than $98 \%$ of the enzymatic activity; and treatment of the plasma with a DPP4-specific inhibitor, sitagliptin, defined sDPP4 as the enzyme likely to be responsible for the in vivo cleavage of CXCL10 (Figure 4A). Due to elevated plasma levels of TIMP-1 (Figure 1A), we did not consider analysis of MMP activity, but normal plasma concentrations of MMP2, MMP9, and MMP12 in our patients support the supposition that DPP4 is the likely in vivo candidate for $\mathrm{NH}_{2}$-terminal cleavage of CXCL10 (Supplemental Figure 2 and data not shown).

Interestingly, when we plotted plasma concentration of CXCL10 and SDPP4 against each other, there was a positive correlation $\left(r_{s}=0.6\right.$, Supplemental Figure $\left.3 \mathrm{~A}\right)$. Both molecules may be expressed by hepatocytes, and chronic inflammation and liver injury may be a common trigger for their expression (Supplemental Figure 3B). A relativity plot of CXCL10, sDPP4, DPP activity, alanine aminotransferase (ALT), and aspartate aminotransferase (AST) indicates such a correlation in chronic HCV patients but not SVRs (Supplemental Figure 3C).
To strengthen our hypothesis, we prepared recombinant CXCL10 (1-77 aa) and CXCL10 (3-77 aa) (Supplemental Figure 4) and evaluated their effect on CXCR3 activity and T cell migration. We performed in vitro studies to confirm that indeed the agonist form, but not the $\mathrm{NH}_{2}$-truncated form, of CXCL10, is capable of (a) directing the migration of $\mathrm{CXCR}^{+}$cells (Figure 5B); (b) mediating receptor internalization in primary PBMCs (data not shown); and (c) triggering a $\mathrm{Ca}^{2+}$ flux in $\mathrm{CHO}$ cells transfected with a CXCR3expressing plasmid (Figure 5C). Finally, we demonstrated that pretreatment with CXCL10 (3-77 aa) antagonized CXCL10 (1-77 aa)-induced $\mathrm{Ca}^{2+}$ mobilization (Figure $5 \mathrm{C}$ ). These data indicate that CXCL10 (1-77 aa) is a DPP4 substrate and that $\mathrm{NH}_{2}$-terminal cleavage results in a competitive antagonist form of CXCL10.

$\mathrm{NH}_{2}$-truncated CXCL10 is the dominant form circulating in chronic HCV patients. The ability to detect agonist and antagonist forms of CXCL10 permitted us to directly test our hypothesis of chemokine antagonism. Due to the fact that DPP4 is bioactive in whole blood (with high levels of activity at room temperature; data not shown), it was necessary that we initiate a new prospective cohort, collecting blood samples using P700 tubes as discussed above. Patient plasma was isolated at the time point prior to treatment initiation, and using the 3-plex Luminex assay (Supplemental Figure 1), we monitored CXCL10 (total), CXCL10 (1-77 aa, referred to as long), and CXCL10 (3-77 aa, referred to as short). Data are presented across 

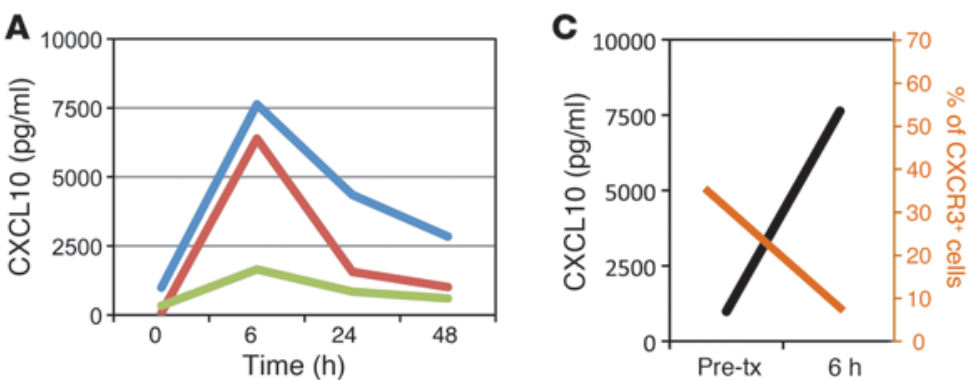

B
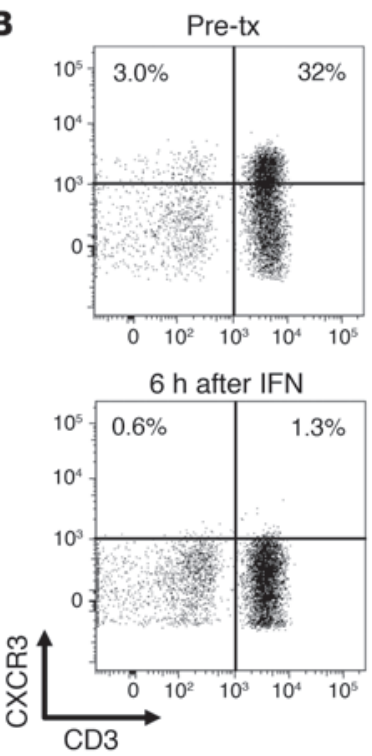

\section{Figure 4}

Induction of agonist CXCL10 results in rapid efflux of $\mathrm{CXCR}^{+} \mathrm{T}$ cells from the circulation. For 5 patients initiating peg-IFN- $\alpha_{2} /$ ribavirin therapy, we monitored the plasma concentration of CXCL10 and the frequency of CXCR3expressing cells. Results for a representative patient are shown. (A) Plasma levels of total CXCL10 (blue line), longform CXCL10 (1-77 aa) (red line), and short-form CXCL10 (3-77 aa) (green line) are shown. (B) CXCR3-expressing $\mathrm{T}$ cells are shown at the time point prior to therapy (pre-tx; time, 0 hours) and 6 hours following initiation of treatment. (C) Total CXCL10 is represented by a black line, permitting comparison with the percentage of circulating CXCR3, represented by the orange line. (D) The absolute cell counts are shown for $\mathrm{CXCR}^{+}$and $\mathrm{CXCR}^{-} \mathrm{T}$ cells. Values shown indicate the percent decrease for the respective populations of cells. two patient groups: those that achieved an early virologic response (EVR) as defined by a 2-log reduction in viral load at week 12 of therapy (Figure 6); and those failing to achieve an EVR (NRs) (Figure 6). Striking, only 10 of 34 patients had detectable levels of agonist CXCL10 (1-77 aa). In contrast, we report detectable levels of antagonist CXCL10 (3-77 aa) in 10 of 21 patients who achieved an EVR and 9 of 13 who were NRs (Figure 6). The plasma concentration of CXCL10 total and antagonist form was higher in NRs as compared with responders (Mann-Whitney $U$ test, $P=0.01$ and 0.04 , respectively). Thus, we conclude that the dominant form of plasma CXCL10 in chronic $\mathrm{HCV}$ patients is the antagonist form and suggest that chemokine antagonism may contribute to treatment failure.

\section{Discussion}

Chemokine antagonism in chronic HCV patients. To our knowledge, our study offers the first evidence for chemokine antagonism as a possible mechanism in HCV disease pathogenesis. Ironically, many of the pieces to this puzzle have been known; however, a synthesis of disparate observations from different fields has not been previously articulated. Several independent studies have shown CXCL10 to be a negative predictive marker for response to therapy in chronic HCV patients (10-13). This observation has held up for genotype 1 and 4 patients (12), including difficult-to-treat patients with high levels of liver fibrosis, coinfected HIV/HCV-infected patients, and individuals with extrahepatic manifestations of HCV chronic infection (e.g., mixed cryoglobulinemia and thyroiditis). Regarding CXCL10 being a substrate for DPP4, the seminal in vitro studies were performed by Proost and Van Damme, who established that CXCL10 (3-77 aa) is capable of acting as a dominant negative, binding CXCR3 without signaling and competitively inhibiting binding by the agonist form of CXCL10 (1-77 aa) (22). DPP4 is constitutively expressed by a wide range of cell types, including hepatocytes, fibroblasts, and epithelial and endothelial cells; and it is an activation marker for lymphocytes, in particular Th1 and effector/memory $\mathrm{CD}^{+} \mathrm{T}$ cells. In addition to cell-associated DPP4, the enzyme is shed via the action of a still-unidentified sheddase, and healthy donors harbor physiologic plasma activity in the range of $12-25 \mathrm{U} / \mathrm{l}$. While published kinetic studies suggest that this should be sufficient to cleave even high concentrations of CXCL10 (34), no prior in vivo studies have confirmed CXCL10 to be present in an $\mathrm{NH}_{2}$-terminus cleaved form. Finally, there have been two clinical reports that have made a link between DPPs and chronic HCV infection. Firneisz et al. noted the link between liver injury and DPP activity and in a large (144 person) cohort reported higher plasma X-prolyl DPP activity in patients with chronic HCV infection than in healthy controls (35). This study, however, did not define the enzyme responsible for the enzymatic activity, nor did it make a link to any of the immunologic or metabolic findings in chronic HCV patients. Yang et al. evaluated sDPP4 as a marker of Th1 cell activation and followed 33 chronic HCV patients, reporting lower sDPP4 plasma concentrations as compared with those in healthy donors (36). Their study, however, did not evaluate enzymatic activity. Thus, we believe our study, through the application of novel tools and a prospective clinical cohort, has offered an integrated analysis, providing an important and unique perspective regarding the inflammatory state of chronic HCV patients. While 
A
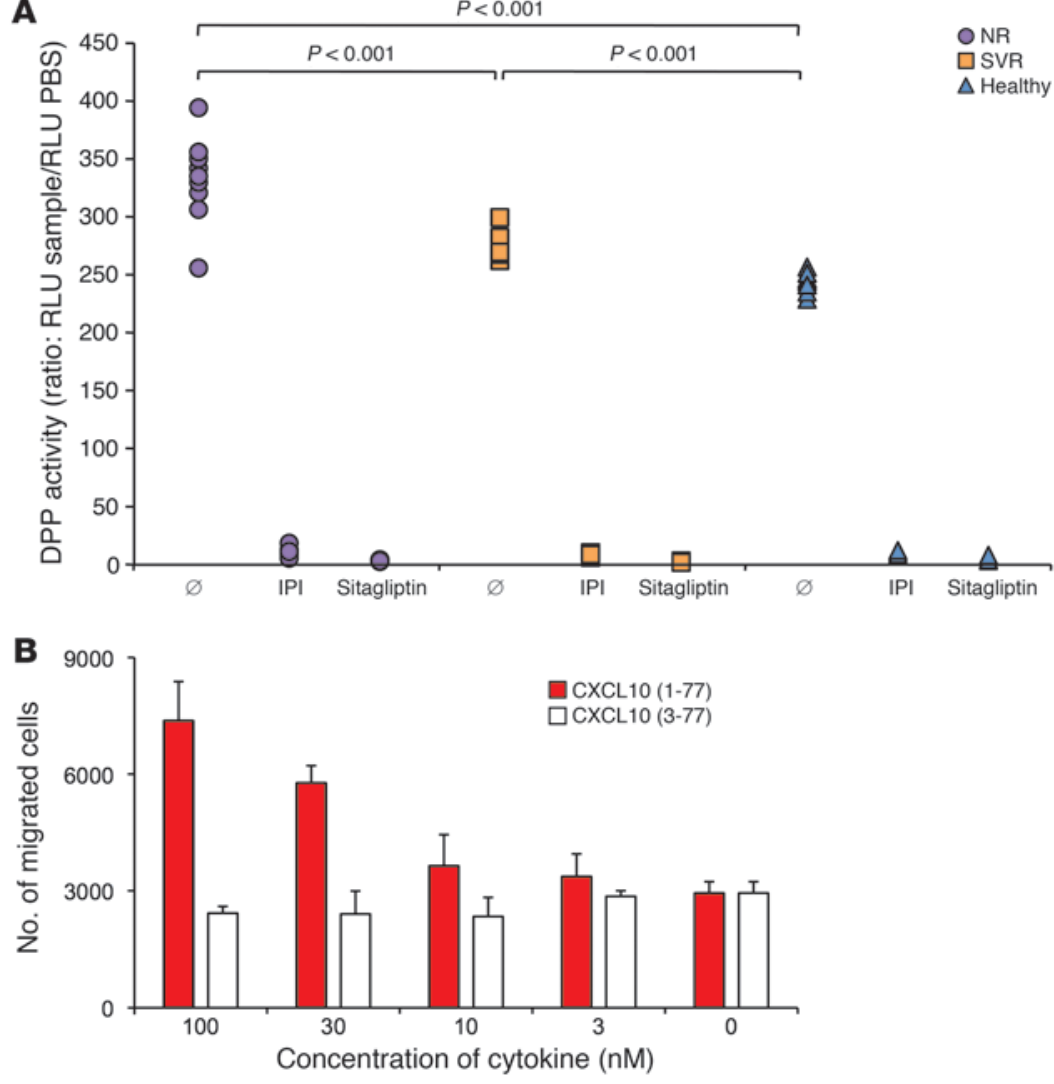

C
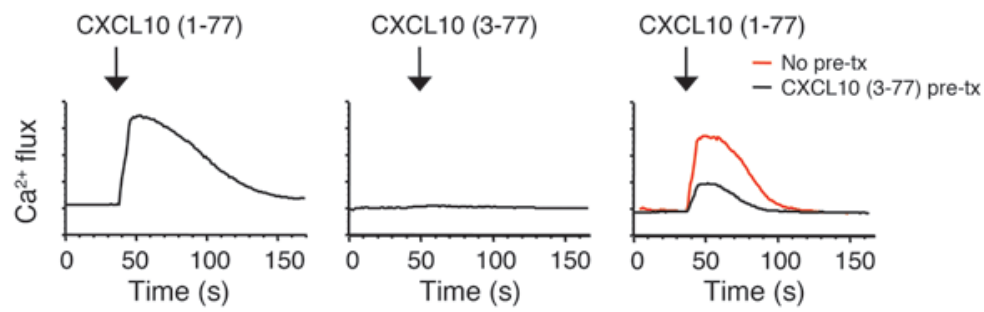

\section{Figure 5}

Elevated X-prolyl DPP activity in chronic HCV patients is mediated by DPP4. (A) The activity of DPP4 was measured using a luciferase-based assay. Plasma from chronic NRs $(n=10)$, SVRs $(n=8)$, and healthy donors $(n=8)$ were tested in the presence or absence of the pan-DPP inhibitor IPI and the DPP4-specific inhibitor sitagliptin. The ratio of RLU of sample/RLU of PBS is represented, with each dot being the average of replicate wells for a single individual. This experiment is representative of 2 independent assays. (B) For in vitro experiments, human recombinant CXCL10 (1-77 aa) was digested in vitro with recombinant DPP4 to obtain CXCL10 (3-77 aa). In migration studies, human PHA-T blasts were prepared as described in Methods. Each bar is the mean of duplicate wells, and error bars indicate 1 SD of the mean. This experiment is representative of results obtained with 3 different healthy donors. (C) $\mathrm{CHO}-\mathrm{K} 1$ cells were stably transfected with a plasmid expressing human CXCR3. Fura-3-AM fluorescent dye-labeled CXCR3-expressing $\mathrm{CHO}$ cells were used for $\mathrm{Ca}^{2+}$ flux experiments. Arrows indicate the injection of the stimulant. In the first plot, $1 \mu \mathrm{M}$ CXCL10 (1-77 aa) was used. In the second plot, $1 \mu \mathrm{M} \mathrm{CXCL10}$ (3-77 aa) was used. And in the third plot, cells were incubated at room temperature for 5 minutes with $1 \mu \mathrm{M}$ CXCL10 (3-77 aa) (pre-tx), followed by a stimulation with $0.2 \mu \mathrm{M}$ CXCL10 (1-77 aa). Data are representative of 3 independent experiments. the demonstration of circulating CXCL10 lacking the penultimate $2 \mathrm{~N}$-terminal amino acids supports a role for DPP4, we could not account for the total amount of CXCL10 detected (total > long + short). As the assays utilize different capture antibodies, it is difficult to directly compare the amount of CXCL10 detected in the different ELISAs. That said, we favor the hypothesis that once cleaved by DPP4, the truncated form of CXCL10 becomes a substrate for other aminopeptidases (e.g., APN or CD13).

DPP4 provides a link between metabolic and immunologic dysregulation in chronic $\mathrm{HCV}$ patients. Interestingly, our observations also point to a link between the metabolic and immunologic perturbations seen in chronic $\mathrm{HCV}$ patients. Insulin resistance and type 2 diabetes are common comorbidities in chronic hepatitis $\mathrm{C}$ patients, with recent estimates indicating that $30 \%-50 \%$ of patients have some degree of metabolic glucose abnormality (37-39). The incretin hormone glucagon-like peptide-1 (GLP-1) has come under close scrutiny as a contributor to insulin resistance, and relevant to our findings, its antagonist activity is due to cleavage by DPP4 (40). While much work will be required to understand this connection at a mechanistic level, it is worthy of mention that insulin resistance is a negative predictor for response to IFN/ribavirin therapy (39,
41). There is also a case report of a diabetic chronic HCV patient who received a 48-week course of IFN/ribavirin and was cured of type 2 diabetes (39). One possible explanation that might account for such a finding is that HCV-driven liver inflammation results in increased DPP4 activity, which in turn catabolized GLP-1 and disrupted glucose homeostasis of the patient. While not currently focused on DPP4, recent clinical studies have aimed at stabilizing patients' glucose control as a means of improving their response to therapy. These initial trials failed (42), but perhaps they were utilizing the wrong anti-hyperglycemic agent(s). Links between type 1 diabetes and CXCL10 have also been reported, with the suggestion that the cell type responsible for expressing the chemokine may favor tolerance versus autoimmunity (43). It may be interesting to reevaluate these studies in light of our findings.

Additional work will be required in order to define how the observation of antagonist plasma CXCL10 compares with the form of CXCL10 found within the liver microenvironment. Indeed, high levels of DPP4 may reflect the high level of tissue remodeling, favoring tissue repair and dampening the high level of liver inflammation. Nonetheless, $\mathrm{T}$ cell infiltration remains high, especially in treatment NRs. Clearly CXCL10 is not the only chemokine 

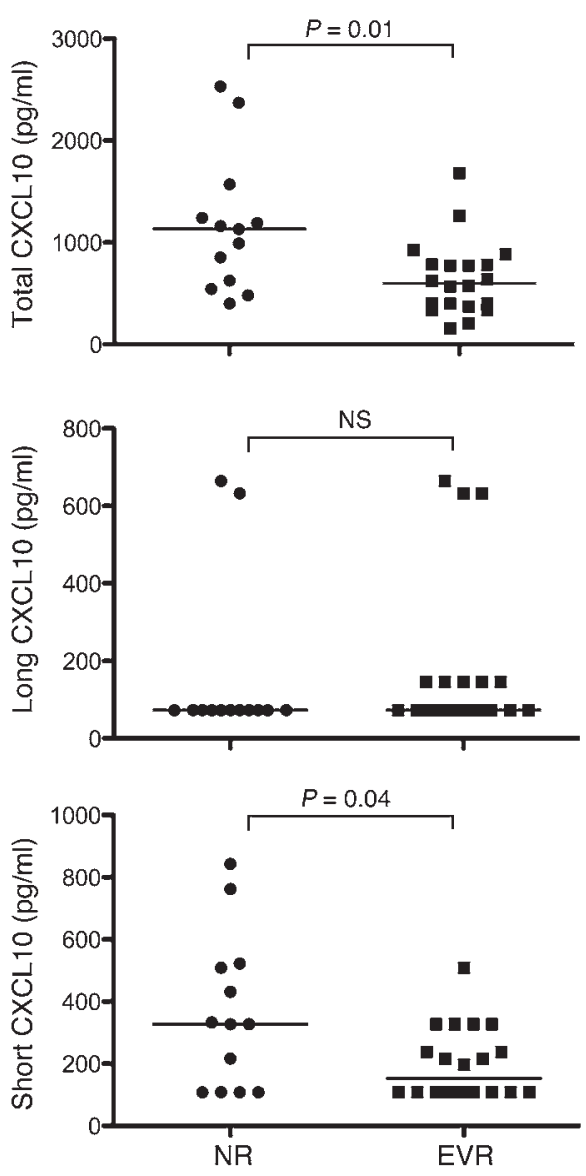

responsible for leukocyte trafficking to the liver, nor are chemokines the only mechanism by which lymphocytes enter the liver parenchyma. An additional challenge will be to define the effects on $\mathrm{HCV}$-specific $\mathrm{T}$ cells versus those that are antigen-nonspecific. Indeed, data from Chisari and colleagues argue that IFN-inducible chemokines mediate antigen-specific mononuclear cell entry into the liver, favoring viral clearance; whereas MMPs are responsible for migration of antigen-nonspecific mononuclear cells, which are responsible for the severity of liver disease (44). While speculative, if these dichotomous mechanisms of $\mathrm{T}$ cell trafficking reflect the pathophysiology of HCV liver inflammation, our data would argue that liver homing of antigen-specific T cells is selectively disrupted by DDP4 cleavage of CXCL10.

In sum, we report the identification of agonist CXCL10 as an in vivo target for DPP4 and the possibility that this cleavage event is linked to treatment failure in chronic HCV patients.

\section{Methods}

\section{Patient samples}

For multi-analyte profiling and MMP2, MMP9, and DPP assays, we obtained banked patient samples from collections performed previously by a member of our research team during the treatment and management of patients with chronic $\mathrm{HCV}$ receiving peg-IFN- $\alpha_{2} /$ ribavirin therapy. All patients had genotype 1 infection. Plasma samples were obtained before, during, and after treatment. Samples were stored at $-80^{\circ} \mathrm{C}$ prior to use. For cellular studies evaluating CXCR3 expression (Figure 2), frozen samples

\section{Figure 6}

The antagonist form of CXCL10 (3-77 aa) is predictive of failure to achieve an EVR. A prospective cohort was initiated and blood sampled in P700 collection tubes at the time point prior to treatment initiation. Thirty-four patients were followed clinically and classified based on their having achieved a 2-log reduction of viral load at week 12 of treatment (referred to as EVRs) or not (referred to as NRs). Plasma separated from the P700 tubes was analyzed using the CXCL10 3-plex. Plasma concentrations of total, long (1-77 aa), and short (3-77 aa) forms of CXCL10 are shown. Each symbol represents an individual patient; the black horizontal bars indicate the median. Mann-Whitney, 2-tailed comparison test was performed between EVRs and NRs, and $P$ values are reported.

were utilized. These samples were obtained as part of study protocol RBM 03-59, approved by the INSERM clinical investigation department, with ethical approval from the Committee for the Protection of Persons (CPP) of Necker Hospital, Paris. HCV patients with chronic infection were identified by anti-HCV antibodies and HCV RNA positivity for more than 6 months. All patients had genotype 1 infection and were treated by a combination of peg-IFN- $\alpha_{2}$ and ribavirin for at least 3 months. Clinical information about patients is reported in Supplemental Table 1. SVRs were defined as individuals absent of HCV RNA for longer than 6 months after termination of therapy. Early virologic responders (EVRs) were defined as individuals achieving a 2-log reduction of virus by 12 weeks of therapy. For analysis of agonist and $\mathrm{NH}_{2}$-truncated forms of CXCL10, new collections were required so that blood samples could be collected in BD P700 tubes (which contain a DPP4 inhibitor), thus preserving the circulating form of CXCL10. In these studies, fresh analysis was performed for CXCR3 expression (Figure 4), and plasma was frozen until used (Figures 4 and 6). These samples were obtained as part of study protocol C07-11 approved by the INSERM clinical investigation department with ethical approval from the CPP Ile-de-France II, Paris).The study protocols conformed to the ethical guidelines of the Declaration of Helsinki, and patients provided informed consent.

\section{Multi-analyte profiling}

Plasma samples were clarified by high-speed centrifugation and analyzed using Luminex xMAP technology. Samples were measured by a diagnostic laboratory, Rules-Based Medicine, for measurement of 83 molecules, with all assays being CLIA certified. The cut-off value was derived by determining the average of more than 200 normals and adding 3 SDs of the mean. The least detectable dose (LDD) is the lower limit at which the system can accurately calculate the concentration of an experimental sample, with confidence that the concentration is higher than that of a blank sample. The LDD is determined by analyzing 20 blank samples, calculating the mean background, and adding 3 SDs of this mean. The lower assay limit (LAL) is each assay's working sensitivity as defined by the lowest concentration calibrator found on the standard curve that provides a quantifiable measurement above background. The LAL is typically lower than the LDD. Analyte measurements reported below the LDD and above the LAL may be real values whose precision is examined closely. Values above the LDD possess excellent precision, with coefficients of variation (CV) less than $10 \%$. For data mining, values below the LAL were replaced with a value that was $50 \%$ of the lowest value measured in the data set. In parallel, analytes not part of this multiplex screen (IFN- $\alpha$ and CXCL10) were analyzed by Luminex, using commercially available kits (Invitrogen). For statistical analysis of biomarker data, nonparametric tests were utilized (Kruskal-Wallis for 3-way tests; and Mann-Whitney for 2-way comparisons). In cases where multiple analyte testing was performed, an FDR correction was imposed. 


\section{Generation of agonist CXCL10 and $\mathrm{NH}_{2}$-truncated CXCL10-specific pAbs}

Production of polyclonal agonist CXCL10-specific antibodies were generated by Proteogenix Co. The $\mathrm{NH}_{2}$-terminal 9 amino acids of the secreted form of CXCL10 (VPLSRTVRC) were synthesized and coupled to keyhole limpet hemocyanin (KLH). Eight New Zealand White rabbits were bled, and preimmune serum was collected before the immunization. Animal immunization and serum isolation were approved by Regierung der Oberpfalz (German veterinarian agency), Regensburg, Germany. After immunization, antisera were tested by direct ELISA (plates were coated with the same peptide used for immunization). After a 90-day immunization protocol consisting of 5 injections, serum IgGs were subjected to an affinity column containing the VPLSRTVRC peptide, then a depletion column containing the short peptide (LSRTVRC, corresponding to $\mathrm{NH}_{2}$-terminal 7 amino acids of DPP4-cleaved CXCL10). Two of the 8 rabbits tested had high affinity and specific reactivity for agonist CXCL10 (1-77 aa). IgG purification was performed and further tested. For data in Figure 3A and the validation of reagents used in Figure 5, we utilized the pAbs isolated from rabbit L3 (and called antiserum L3), which after immunodepletion showed no binding to CXCL10 (3-77 aa) (up to concentrations of $2 \mu \mathrm{g} / \mathrm{ml}$ ) and had a limit of detection of $125 \mathrm{pg} / \mathrm{ml}$ for CXCL10 (1-77 aa) in a sandwich ELISA. Additionally, we demonstrated lack of cross-reactivity with other $\alpha$-chemokines (data not shown). Production of monoclonal single-chain antibodies specific for the agonist form and also the neo-epitope created by DPP4 cleavage of CXCL10 were generated by AbD Serotec. Briefly, peptide corresponding to the $\mathrm{NH}_{2}$-terminal 7 amino acids of DPP4-cleaved CXCL10 (LSRTVRC) and peptide corresponding to the native protein (VPLSRTVRC) were conjugated to BSA and TRF. The Human Combinatorial Antibody (HuCal) Library was screened first with the conjugated peptides coated in the ELISA plate; clones reactive for the neo-epitope and not the native peptide were then screened with the recombinant proteins (CXCL10 [3-77 aa] and [1-77 aa]). Clone 10.1 is specific for the $\mathrm{N}$-terminus of the full-length form of CXCL10; and clone 52.1 is specific for the neo-epitope present in the DPP4-cleaved antagonist form of CXCL10. These two antibodies along with a commercially available clone 33036 (R\&D Systems) were utilized for the generation of a Luminex 3-plex. This assay was CLIA certified, and standard curves are shown in Supplemental Figure 1.

\section{Assays for discriminating between agonist CXCL10 and $\mathrm{NH}_{2}$-truncated $\mathrm{CXCL10}$}

To generate CXCL10 (3-77 aa), recombinant human CXCL10 (Peprotech) was incubated with recombinant DPP4 (Sigma-Aldrich) in a 100-mM Tris$\mathrm{HCl}, \mathrm{pH} 8$, solution for 2 hours at $37^{\circ} \mathrm{C}$.

SELDI-TOF mass spectrometry. For surface-enhanced laser desorption/ionization-time-of-flight (SELDI-TOF) mass spectrometry, $1 \mu$ l of the digested product was spotted onto an $\mathrm{H} 4$ protein chip. Arrays were incubated and washed as per the manufacturer's instructions and analyzed using the ProteinChip System Series 4000 (Ciphergen). The range of MWs investigated was $0-20,000 \mathrm{Da}$. The focus mass was set at $8.5 \mathrm{kDa}$. Data were analyzed using CiphergenExpress Software.

Chemotaxis assays. PBMCs were purified from healthy volunteers and treated with $1 \mathrm{mg} / \mathrm{ml}$ phytohemagglutinin for 3 days, then $40 \mathrm{U} / \mathrm{ml}$ of recombinant hIL-2 (R\&D Systems) was added for and additional 2 days prior to use of the cells. Lymphocyte chemotaxis was performed using 5 - $\mu \mathrm{m}$-pore-size Multiscreen-MIC plates (Millipore). To monitor CXCL10 (1-77 aa)- or CXCL10 (3-77 aa)-directed migration, we added lymphocytes to the top chamber and chemokine to the bottom. Lymphocytes were allowed to migrate for 2 hours at $37^{\circ} \mathrm{C}$, and cells in the bottom chamber were counted using a Guava cytometer (Guava Technologies).
Calcium flux assays. Increased intracellular $\left[\mathrm{Ca}^{2+}\right]$ in response to CXCR3 signaling was monitored by FACS. Briefly, CHO-K1 cells were transfected with a plasmid encoding the full-length open reading frame of CXCR3 (provided by C.P. Tensen, Leiden University Medical Center [LUMC], Leiden, The Netherlands). Stably transfected cells were screened for CXCR3 expression (anti-human CXCR3 clone 1C6, BD) and loaded with Fura-3-AM. $\mathrm{Ca}^{2+}$ release was measured using a Canto II Cytometer. Baseline signal was determined prior to addition of chemokine. For competitive antagonist assessment, cells were first incubated for 5 minutes with $1.0 \mu \mathrm{M}$ CXCL10 (3-77 aa), then exposed to $0.2 \mu \mathrm{M}$ CXCL10 (1-77 aa).

Sandwich ELISA. Maxisorp plates (Nunc) were coated with $2 \mu \mathrm{g} / \mathrm{ml}$ of the capture antibody mAb anti-CXCL10 (clone 33036) in PBS overnight at $4{ }^{\circ} \mathrm{C}$. The wells were saturated with $200 \mu 10.05 \%$ Tween-20, $1 \%$ BSA in PBS for 1-2 hours at room temperature. Samples were diluted in saturation buffer and incubated at room temperature for 2 hours. A standard curve was obtained by diluting recombinant CXCL10 (1-77 aa) or CXCL10 (3-77 aa). Each experiment was run in duplicate with two different detection antibodies - a commercially available goat $\mathrm{pAb}$ that recognized both forms of CXCL10 and a rabbit pAb (antiserum L3) that is specific for the agonist form of CXCL10. For detection of the 2 -amino-acid $\mathrm{NH}_{2}$-truncated form of CXCL10, we utilized the single-chain $\mathrm{Ab}$ (clone 52.1) as the capture reagent and goat $\mathrm{pAb}$ anti-CXCL10 for detection. Plates were read in a Labsystems Multiskan MS (Thermo) device.

CXCL10 3-plex Luminex assay. The CXCL10 3-plex is a microspherebased assay and consists of using antigen-specific antibodies and other optimized reagents in a capture-sandwich format. Fluorescent beads of distinct color were conjugated with mAb 33036 (R\&D Systems), clone 10.1, or clone 52.1, respectively. All incubations took place at room temperature. Five microliters of a diluted mixture of capture-antibody microspheres were mixed with $5 \mu \mathrm{l}$ blocker and $10 \mu \mathrm{l}$ standard, prediluted sample or control in a hard-bottom microtiter plate. Samples were prediluted 1:5. The plate was incubated for 1 hour. Ten microliters biotinylated detection antibody, a polyclonal goat anti-CXCL10 (R\&D Systems), was added to each well, thoroughly mixed, and incubated for 1 hour. Ten microliters diluted streptavidin-phycoerythrin was added to each well, thoroughly mixed, and incubated for 60 minutes. The reaction contents of the hard-bottom plate were then transferred to the respective wells of the filter plate. All wells were vacuum-aspirated, and the contents were washed twice with $100 \mu \mathrm{l}$ wash buffer. After the last wash, $100 \mu \mathrm{l}$ wash buffer was added to each well, and the washed microspheres were resuspended with thorough mixing. The plate was then analyzed on the Luminex 100 Analyzer. Standard curves for the three assays are shown. Biologic CXCL10 produced by culturing PBMCs with IFN- $\alpha_{2}$ was used for the standard for total and CXCL10 (1-77 aa) assays. Recombinant protein cleaved by DPP4 was used for the CXCL10 (3-77 aa) assay. The activity of DPP4 was measured using a luciferase based assay - DPP4-Glo protease Assay (Promega).

\section{Statistics}

A Mann-Whitney $U$ test was used to determine whether a difference existed between 2 groups of individuals. A 2-tailed comparison was performed in all cases. A $P$ value less than 0.05 was considered to be statistically significant. Kruskal-Wallis 1-way ANOVA, a nonparametric method for testing equality of population medians among groups, was used for Figure 1A. An FDR-corrected $P$ value of less than 0.05 was considered to be statistically significant.

\section{Acknowledgments}

The authors would like to thank the INSERM medical affairs team, the members of the Cochin Hepatology Unit, and Stéphanie 
Thomas for assistance in patient recruitment and sample collections. We appreciate the assistance of Jacques D'Alayer for the mass spectrometry analysis. We also thank Sebastian Amigorena and Philippe Bousso for their thoughtful comments and suggestions for the manuscript. James Mapes is a cofounder of Rules-Based Medicine Inc., a biotech company that was involved in developing the CXCL10 3-plex. Finally, we acknowledge the generous participation of the patients who agreed to be part of our studies. This work was supported in part by grants from the Agence Nationale de Recherche sur le Sida et les hépatites virales (ANRS) and the European Research Council (ERC).

Received for publication August 17, 2010, and accepted in revised form October 6, 2010.

Address correspondence to: Matthew L. Albert, Institut Pasteur 25, Rue du Dr. Roux, Paris, France 75724. Phone: 33.0.1.45.68.85.45; Fax: 33.0.1.45.68.85.48; E-mail: albertm@pasteur.fr.
1. Houghton M, Abrignani S. Prospects for a vaccine against the hepatitis C virus. Nature. 2005; 436(7053):961-966.

2. Bowen DG, Walker CM. Adaptive immune responses in acute and chronic hepatitis $C$ virus infection. Nature. 2005;436(7053):946-952.

3. Cooper S, et al. Analysis of a successful immune response against hepatitis C virus. Immunity. 1999; 10(4):439-449.

4. Lechner F, et al. Analysis of successful immune responses in persons infected with hepatitis C virus. J Exp Med. 2000;191(9):1499-1512.

5. Khakoo SI, et al. HLA and NK cell inhibitory receptor genes in resolving hepatitis $\mathrm{C}$ virus infection. Science. 2004;305(5685):872-874.

6. Feld JJ, Hoofnagle JH. Mechanism of action of interferon and ribavirin in treatment of hepatitis C. Nature. 2005;436(7053):967-972.

7. Luster AD, Ravetch JV. Biochemical characterization of a gamma interferon-inducible cytokine (IP-10). J Exp Med. 1987;166(4):1084-1097.

8. Singh UP, Singh S, Taub DD, Lillard JW Jr. Inhibition of IFN-gamma-inducible protein-10 abrogates colitis in IL-10-/- mice. J Immunol. 2003;171(3):1401-1406.

9. Rhode A, et al. Islet-specific expression of CXCL10 causes spontaneous islet infiltration and accelerates diabetes development. J Immunol. 2005; 175(6):3516-3524.

10. Butera D, et al. Plasma chemokine levels correlate with the outcome of antiviral therapy in patients with hepatitis C. Blood. 2005;106(4):1175-1182.

11. Lagging $M$, et al. IP-10 predicts viral response and therapeutic outcome in difficult-to-treat patients with HCV genotype 1 infection. Hepatology. 2006; 44(6):1617-1625.

12. Romero AI, et al. Interferon (IFN)-gamma-inducible protein-10: association with histological results, viral kinetics, and outcome during treatment with pegylated IFN-alpha $2 \mathrm{a}$ and ribavirin for chronic hepatitis C virus infection. J Infect Dis. 2006; 194(7):895-903.

13. Diago M, et al. Association of pretreatment serum interferon gamma inducible protein 10 levels with sustained virological response to peginterferon plus ribavirin therapy in genotype 1 infected patients with chronic hepatitis C. Gut. 2006;55(3):374-379.

14. Roe B, et al. Elevated serum levels of interferongamma -inducible protein-10 in patients coinfected with hepatitis C virus and HIV.J Infect Dis. 2007; 196(7):1053-1057.

15. Zeremski M, Markatou M, Brown QB, Dorante G, Cunningham-Rundles S, Talal AH. Interferon gamma-inducible protein 10: a predictive marker of successful treatment response in hepatitis C virus/HIV-coinfected patients. J Acquir Immune Defic Syndr. 2007;45(3):262-268.

16. Antonelli A, et al. Alpha-chemokine CXCL10 and beta-chemokine CCL2 serum levels in patients with hepatitis $\mathrm{C}$-associated cryoglobulinemia in the presence or absence of autoimmune thyroiditis. Metabolism. 2008;57(9):1270-1277.

17. Schwartz TW, Frimurer TM, Holst B, Rosenkilde MM, Elling CE. Molecular mechanism of 7TM receptor activation - a global toggle switch model. Annu Rev Pharmacol Toxicol. 2006;46:481-519.

18. Van den Steen PE, Husson SJ, Proost P, Van Damme J, Opdenakker G. Carboxyterminal cleavage of the chemokines MIG and IP-10 by gelatinase B and neutrophil collagenase. Biochem Biophys Res Commun. 2003;310(3):889-896.

19. Loos T, et al. Citrullination of CXCL10 and CXCL11 by peptidylarginine deiminase: a naturally occurring posttranslational modification of chemokines and new dimension of immunoregulation. Blood. 2008;112(7):2648-2656

20. Gorrell MD. Dipeptidyl peptidase IV and related enzymes in cell biology and liver disorders. Clin Sci (Lond). 2005;108(4):277-292.

21. Ludwig A, Schiemann F, Mentlein R, Lindner B, Brandt E. Dipeptidyl peptidase IV (CD26) on T cells cleaves the CXC chemokine CXCL11 (I-TAC) and abolishes the stimulating but not the desensitizing potential of the chemokine. J Lenkoc Biol. 2002; 72(1):183-191.

22. Proost $\mathrm{P}$, et al. Amino-terminal truncation of CXCR3 agonists impairs receptor signaling and lymphocyte chemotaxis, while preserving antiangiogenic properties. Blood. 2001;98(13):3554-3561.

23. McQuibban GA, Gong JH, Tam EM, McCulloch CA, Clark-Lewis I, Overall CM. Inflammation dampened by gelatinase A cleavage of monocyte chemoattractant protein-3. Science. 2000;289(5482):1202-1206.

24. Zhang K, et al. HIV-induced metalloproteinase processing of the chemokine stromal cell derived factor-1 causes neurodegeneration. Nat Neurosci. 2003; 6(10):1064-1071.

25. Dinarello CA. Interleukin-18, a proinflammatory cytokine. Eur Cytokine Netw. 2000;11(3):483-486.

26. Kaplanski G, Farnarier C, Payan MJ, Bongrand P, Durand JM. Increased levels of soluble adhesion molecules in the serum of patients with hepatitis C. Correlation with cytokine concentrations and liver inflammation and fibrosis. Dig Dis Sci. 1997; 42(11):2277-2284.

27. Zaman A, Rosen HR, Ingram K, Corless CL, Oh E, Smith K. Assessment of FIBROSpect II to detect hepatic fibrosis in chronic hepatitis $\mathrm{C}$ patients. Am JMed. 2007;120(3):280.e9-280.e14.

28. Thimme R, et al. Viral and immunological determinants of hepatitis $C$ virus clearance, persistence, and disease. Proc Natl Acad Sci U S A. 2002; 99(24):15661-15668.

29. Boker KH, Pehle B, Steinmetz C, Breitenstein K, Bahr M, Lichtinghagen R. Tissue inhibitors of metalloproteinases in liver and serum/plasma in chronic active hepatitis $\mathrm{C}$ and $\mathrm{HCV}$-induced cirrho- sis. Hepatogastroenterology. 2000;47(33):812-819.

30. Zeremski M, et al. Intrahepatic levels of CXCR3associated chemokines correlate with liver inflammation and fibrosis in chronic hepatitis C. Hepatology. 2008;48(5):1440-1450.

31. Ajami K, et al. Stromal cell-derived factors 1alpha and 1 beta, inflammatory protein-10 and interferon-inducible $T$ cell chemo-attractant are novel substrates of dipeptidyl peptidase 8. FEBS Lett. 2008; 582(5):819-825.

32. Bauvois B. Transmembrane proteases in cell growth and invasion: new contributors to angiogenesis? Oncogene. 2004;23(2):317-329.

33. Cox JH, Dean RA, Roberts CR, Overall CM. Matrix metalloproteinase processing of CXCL11/I-TAC results in loss of chemoattractant activity and altered glycosaminoglycan binding. J Biol Chem. 2008; 283(28):19389-19399.

34. Lambeir AM, et al. Kinetic investigation of chemokine truncation by CD26/dipeptidyl peptidase IV reveals a striking selectivity within the chemokine family. J Biol Chem. 2001;276(32):29839-29845.

35. Firneisz G, Lakatos PL, Szalay F. Serum dipeptidyl peptidase IV (DPP IV, CD26) activity in chronic hepatitis C. Scand J Gastroenterol. 2001;36(8):877-880.

36. Yang SS, Fu LS, Chang CS, Yeh HZ, Chen GH, Kao JH. Changes of soluble CD26 and CD30 levels correlate with response to interferon plus ribavirin therapy in patients with chronic hepatitis C. J Gastroenterol Hepatol. 2006;21(12):1789-1793.

37. D'Souza R, Sabin CA, Foster GR. Insulin resistance plays a significant role in liver fibrosis in chronic hepatitis $\mathrm{C}$ and in the response to antiviral therapy. Am J Gastroenterol. 2005;100(7):1509-1515.

38. Romero-Gomez M, et al. Insulin resistance impairs sustained response rate to peginterferon plus ribavirin in chronic hepatitis $C$ patients. Gastroenterology. 2005;128(3):636-641.

39. Romero-Gomez M. Insulin resistance and hepatitis C. World J Gastroenterol. 2006;12(44):7075-7080.

40. Chowdhury TA, Hossain B. New drugs for the treatment of type 2 diabetes. BrJ Hosp Med (Lond). 2007; 68(4):178-183.

41. Negro F. Insulin resistance and HCV: will new knowledge modify clinical management? J Hepatol. 2006;45(4):514-519.

42. Romero-Gomez M, et al. Treatment of insulin resistance with metformin in naive genotype 1 chronic hepatitis $\mathrm{C}$ patients receiving peginterferon alfa-2a plus ribavirin. Hepatology. 2009;50(6):1702-1708.

43. Christen U, Von Herrath MG. IP-10 and type 1 diabetes: a question of time and location. Autoimmunity. 2004;37(4):273-282.

44. Sitia G, Isogawa M, Iannacone M, Campbell IL, Chisari FV, Guidotti LG. MMPs are required for recruitment of antigen-nonspecific mononuclear cells into the liver by CTLs. J Clin Invest. 2004; 113(8):1158-1167. 